\title{
Imperforate hymen presenting as acute urinary retention in an adolescent girl
}

\author{
Renu Prabha $^{1 *}$, Santosh Kumar ${ }^{2}$, Saumyajyoti Kundu ${ }^{1}$ \\ ${ }^{1}$ Department of Obstetrics and Gynecology, ${ }^{2}$ Department of Pediatrics, MGM Medical College and LSK Hospital, \\ Kishanganj, Bihar, India \\ Received: 21 September 2016 \\ Accepted: 18 October 2016 \\ *Correspondence: \\ Dr. Renu Prabha, \\ E-mail: drrenuprabha@gmail.com \\ Copyright: (c) the author(s), publisher and licensee Medip Academy. This is an open-access article distributed under \\ the terms of the Creative Commons Attribution Non-Commercial License, which permits unrestricted non-commercial \\ use, distribution, and reproduction in any medium, provided the original work is properly cited.
}

\begin{abstract}
Acute urinary retention in a female child is rare because of its anatomy. Haematocolpos can cause a mechanical obstruction, resulting in acute urinary retention. We are presenting a case report of 13 year old female presented with complain of urine retention for 24 hours. She had no significant medical history and she had not attained menarche. Ultrasound abdomen showed hematocolpos like mass compressing the bladder. On examination; she had a palpable bladder and a bluish-grey bulge posterior to her urethral meatus. She underwent a hymenotomy using cruciate incision with drainage of the hematocolpos. Postoperatively, she made a good recovery with a successful removal of the urinary catheter. She has started normal menses and had no further urinary problems. We emphasise to include hematocolpos, even though uncommon, in differential diagnosis and should be ruled out in adolescent females with amenorrhoea and acute urinary retention.
\end{abstract}

Keywords: Acute urinary retention, Adolescence, Hematocolpos, Imperforate hymen

\section{INTRODUCTION}

Urinary retention is a rare symptom in young girls. Urinary retention could be caused by mechanical obstruction to the bladder especially in adolescent girls. Haematocolpos, where the vagina is filled with menstrual blood, often caused due to an imperforate hymen, can led to mechanical obstruction to the bladder resulting in urinary retention. ${ }^{1}$

\section{CASE REPORT}

A 13-year-old girl presented to the pediatric emergency department with a one-day history of acute urinary retention associated with suprapubic pain. She had no history of abdominal trauma. There was no history of vomiting, jaundice, pedal edema or hematuria. She had not attained her menarche but she had history of cyclical abdominal cramping pains for the last 10 months. On examination, her abdomen was moderately distended with mild suprapubical tenderness. Her bladder was palpable. On her external genitalia examination, she was noted to have a nontender bluish-grey bulge posterior to the urethra. Neurological examination was normal. Her renal functions tests were normal and urinary pregnancy test was negative. Urinary bladder catheterization led to passage of $1200 \mathrm{ml}$ of urine that relieved her of suprapubic pain. An ultrasound scan of the lower abdomen showed a hematocolpos like mass having dimensions of $12 \times 10 \times 10 \mathrm{~cm}$ lying posterior to the bladder, inseparable from and lying immediately inferior to the uterus. No other abnormal findings were detected. Appearances were consistent with a hugely distended uterus filled with menstrual products. Gynecological opinion was sought and subsequently she underwent a hymenotomy using a cruciate incision with drainage of hematocolpos (Figure 1).

She had no urinary complains postoperatively and thereafter. She attained her menarche one month later and regular menstrual cycles thereafter. 


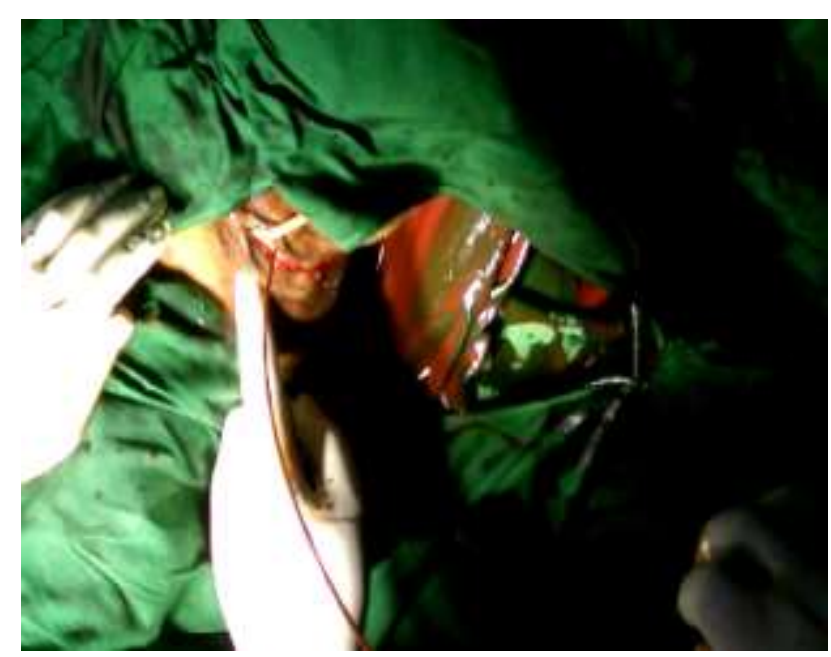

Figure 1: Imperforate hymen with hematocolpos.

\section{DISCUSSION}

Acute urinary retention in children is a rare especially in females. It is defined as the inability to void for over 12 h. Causes of acute urinary retention in children is classified as primary and secondary. Primary causes are urinary tract infection, constipation, neurological dysfunction. Secondary causes are; bladder neoplasm, focal inflammatory reaction, incarcerated inguinal hernia, haematocolpos and pelvic masses. ${ }^{1}$

Imperforate hymen is a rare genital tract anomaly which has an incidence of about 1 in 2000. ${ }^{1}$ The frequency of imperforate hymen with hematocolpos is reported as $0.14 \%$ and approximately fifty percent presents with urinary retention. ${ }^{2,3}$ Urinary retention presents secondary to the haematocolpos due to mechanical compression of the urethra caused by pressure on the posterior wall of the bladder. ${ }^{1}$ Management of imperforate hymen with hematocolpos includes catheterisation of the bladder in cases of retention and then a cruciate hymenectomy under general anaesthesia with evacuation of menstrual products. Most of the patients are discharged soon after the procedure with no postoperative complications. This case report emphasizes to include hematocolpos, even though uncommon, in differential diagnosis and should be ruled out in peripubertal females with amenorrhoea and acute urinary retention.

\section{Funding: No funding sources \\ Conflict of interest: None declared \\ Ethical approval: Not required}

\section{REFERENCES}

1. Chang JW, Yang LY, Wang HH. Acute urinary retention as the presentation of imperforate hymen. $\mathrm{J}$ Chin Med Assoc. 2007;2013:559-61.

2. Mahmood M, Ahzan S, Zaidi Z. Haematocolpos as a cause of urinary retention in the young female. J Pak Med Assoc. 2001;2013:298-9.

3. Ercan CM, Karasahin KE, Alanbay I. Imperforate hymen causing hematocolpos and acute urinary retention in an adolescent girl. Taiwan $\mathrm{J}$ Obstet Gynecol. 2011;2013:118-20.

Cite this article as: Prabha R, Kumar S, Kundu S. Imperforate hymen presenting as acute urinary retention in an adolescent girl. Int J Reprod Contracept Obstet Gynecol 2016;5:4484-5. 